\title{
METCAM/MUCI 8 plays a novel tumor and metastasis suppressor role in the progression of human ovarian cancer cells
}

\begin{abstract}
METCAM/MUC18, an integral membrane cell adhesion molecule (CAM) in the Ig-like gene super-family, is capable of performing typical functions of CAMs, such as mediating cell-cell and cell-extracellular interactions, crosstalk with intracellular signaling pathways, and modulating social behaviors of cells. The role of METCAM/MUC18 in the progression of ovarian cancer cells has not been well studied. Previous studies showed that METCAM/ MUC18 is expressed in normal ovarian epithelial cells, but expressed at higher levels in ovarian cancer tissues, suggesting that METCAM/MUC18 may serve as a biomarker for the malignant progression of clinical ovarian cancers and it has been implicated for playing a positive role in the progression of the cancer. Recently we provided evidence from in vitro and in vivo studies to suggest that the above notion is a fortuitous correlation and that METCAM/MUC18 actually serves as a tumor and metastasis suppressor for the malignant progression of ovarian cancer cells, similar to its role in the malignant progression of one mouse melanoma cell line and nasopharyngeal carcinoma type I. Many possible mechanisms mediated by this CAM during early tumor development and metastasis are suggested. Furthermore, we suggest that METCAM/MUC18 may be used a therapeutic reagent to arrest the malignant progression of clinical ovarian cancer.
\end{abstract}

Keywords: Human METCAM/MUC18 expression, Ovarian cells, SC \& IP injections, Tumorigenesis and progression, Athymic nude mice
Volume 6 Issue 4 - 2017

\author{
Guang-Jer Wu $\mathbf{u}^{1,2}$ \\ 'Department of Microbiology and Immunology, Emory \\ University School of Medicine, USA \\ ${ }^{2}$ Department of Bioscience Technology and Center for \\ Biomedical Technology, Chung Yuan Christian University, Taiwan
}

Correspondence: Guang-Jer Wu, Department of Bioscience Technology, Chung Yuan Christian University, Chung Li, Taiwan 32023, Tel 886-3-265-3507, Fax 886-3-265-3599,

Email guangj.wu@gmail.com, gjwu@cycu.edu.tw

Received: January 14, 2017 | Published: March 21, 2017
Abbreviations: CAM, Cell Adhesion Molecule; huMETCAM/ MUC18, human METCAM/MUC18; METCAM, Metastasis Cell Adhesion Molecule; $I P$, Intra-Peritoneal; SC, Subcutaneous

\section{Introduction: current status of ovarian cancer}

Epithelial ovarian cancer (EOC) is the fifth leading cause of female cancers in USA with a high fatality rate of about $65 \%{ }^{1}$ The high lethality of the cancer is because the early stage of the disease is mostly asymptomatic and therefore remains undiagnosed until the cancer has already disseminated throughout the peritoneal cavity (at clinical stages of III and IV). ${ }^{2}$ The early stage disease can be treated successfully with a five-year survival rate of more than $90 \%$, however, effective therapy for the advanced-stage disease is lacking because of the strong chemo-resistance of recurrent ovarian cancer. ${ }^{2}$

The major challenges for combating ovarian cancer are:

a. The ovarian cancer is histologically and molecularly heterogeneous with at least four major subtypes, such as serous adenocarcinoma (75\%), endometrioid adenocarcinoma (10\%), mucinous adenocarcinoma (3\%), and clear cell carcinomas $(5$ $10 \%),{ }^{3,4}$

b. There is a lack of reliable specific diagnostic markers for an effective early diagnosis of each subtype, though molecular signatures of the major subtypes are available, ${ }^{5}$ and

c. Very little is known of how ovarian tumor emerges and how it progresses to malignancy. ${ }^{6}$

\section{Cell adhesion molecules and the progression of ovarian cancer}

In general, tumorigenesis is a complex process involving changes of several biological characteristics, ${ }^{7}$ including the aberrant expression of cell adhesion molecules (CAMs). ${ }^{7}$ CAMs play many important physiological functions, such as organ formation, tissue architecture, vascularization and angiogenesis, immune response, inflammation, wound healing, and cellular social behaviors. ${ }^{8}$ Since CAMs govern the social behaviors of cells by affecting the adhesion status of cells and cross-talk and modulating intracellular signal transduction pathways, they also play an important function in cancer cell metastasis. This is because tumor progression is induced by a complex cross-talk between tumor cells and stromal cells in the surrounding tissues. ${ }^{9}$ These interactions are, at least in part, mediated by cell adhesion molecules (CAMs) ${ }^{7-9}$ Thus the altered expression of CAMs can change motility and invasiveness, affect survival and growth of tumor cells, and alter angiogenesis. ${ }^{7-9}$ As such, CAMs may promote or suppress the metastatic potential of tumor cells. ${ }^{10}$ Similar to other epithelial cancers, cell adhesion molecules must play a role in the progression of ovarian cancer, especially since aberrant expression of various CAMs, such as mucins, ${ }^{11}$ integrins, ${ }^{12} \mathrm{CD} 44,{ }^{13} \mathrm{~L} 1 \mathrm{CAM},{ }^{14}$ cadherin,${ }^{15}$ claudins, ${ }^{16}$ EpCAM,${ }^{17}$ ALCAM $^{18}$ and METCAM/ MUC18, ${ }^{19,20}$ has been associated with the malignant progression of ovarian cancer. Some of the CAMs may play a positive role, such as MUC4, ${ }^{21} \mathrm{CD} 44,{ }^{22} \mathrm{~L} 1 \mathrm{CAM},{ }^{23}$ ALCAM, ${ }^{18}$ and P-cadherin ${ }^{24}$; however, some a negative role, such as b3-integrin, ${ }^{25}$ E-cadherin, ${ }^{26}$ claudin-3, $4, \& 7,{ }^{27} \mathrm{EpCAM}^{28}$ and $\mathrm{KAI} 1,{ }^{29}$ in the progression of ovarian cancer cells. We have been focusing our studies on the role of METCAM/ MUC18 in the progression of several epithelial tumors, including epithelial ovarian tumors. ${ }^{30}$

\section{The role of METCAM/MUCI 8 in the progression of epithelial cancers}

Human METCAM/MUC18 (or MCAM, Mel-CAM, S-endo1, CD146, or A32), an integral membrane cell adhesion molecule (CAM) in the Ig-like gene superfamily, has an N-terminal extra-cellular domain of 558 amino acids, a transmembrane domain, and a short 
intra-cellular cytoplasmic domain (64 amino acids) at the C-terminus, as shown in the following Figure $1.30,31$

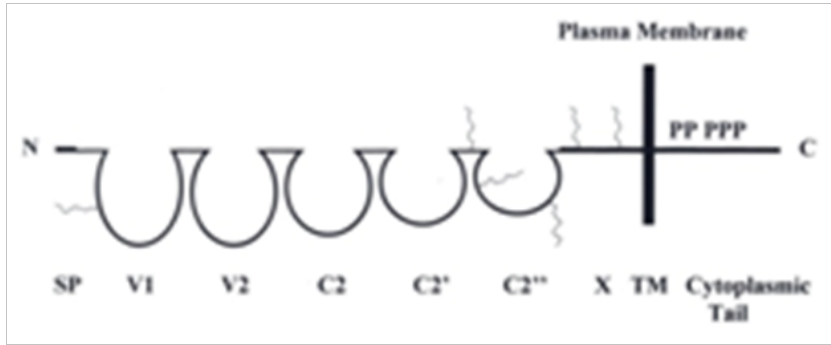

Figure I HuMETCAM protein structure.SP stands for signal peptide sequence, VI,V2, C2, C2', C2" for five lg-like domains (each held by a disulfide bond) and $X$ for one domain (without any disulfide bond) in the extracellular region, and TM for transmembrane domain. P stands for five potential phosphorylation sites (one for PKA, three for PKC, and one for CK2) in the cytoplasmic tail. The six conserved $\mathrm{N}$-glycosylation sites are shown as wiggled lines in the extracellular domains of $\mathrm{VI}$, between $\mathrm{C2}$ ' and C2", C2", and X.

As shown in the Figure 1, the extra-cellular domain of the protein comprises a signal peptide sequence and five immunoglobulin-like domains and one $\mathrm{X}$ domain. ${ }^{30,31}$ The cytoplasmic domain contains five consensus sequences potentially to be phosphorylated by PKA, PKC, and CK2. ${ }^{30,31}$ Thus human METCAM/MUC18 is capable of performing typical functions of CAMs, such as governing the social behaviors by affecting the adhesion status of cells and modulating cell signaling. Therefore, an altered expression of METCAM/MUC18 may affect motility and invasiveness of many tumor cells in vitro and tumorigenesis and metastasis in vivo. ${ }^{30}$

Human METCAM/MUC18 is only expressed in several normal tissues, such as hair follicular cells, smooth muscle cells, endothelial cells, cerebellum, basal cells of the lung, activated T cells, intermediate trophoblasts, ${ }^{32}$ normal breast epithelium, ${ }^{33}$ ovarian epithelium, ${ }^{20}$ and nasopharyngeal epithelium. ${ }^{34}$ Human METCAM/MUC18 is also expressed in several epithelial tumors, such as melanoma, prostate cancer, osteosarcoma, breast carcinoma, and intermediate trophoblast tumors. $^{30,32}$ Over-expression of METCAM/MUC18 promotes the tumorigenesis of prostate cancer ${ }^{35}$ and breast carcinoma, ${ }^{36,37}$ but it has a minimal effect on the tumorigenesis of melanoma. ${ }^{38}$ Over-expression of METCAM/MUC18 also initiates the metastasis of prostate cancer $^{39}$ and promotes the metastasis of melanoma ${ }^{30,38}$ and breast carcinoma. ${ }^{40}$

On the contrary, the possibility that the over-expression of METCAM/MUC18 might play a tumor suppressor role was first suggested by Shih et al. $^{33}$ who found that METCAM/MUC18 expression suppressed tumorigenesis of a breast cancer cell line MCF-7 in SCID mice. However, this notion was revoked by recently published evidence, which supported the positive role of METCAM/ MUC18 in the progression of breast cancer cells, ${ }^{36,37,40}$ similar to its role in the progression of melanoma and prostate cancer cells. The role of METCAM/MUC18 in the progression of ovarian cancer has not been well studied. The following section describes the recent findings for the role of METCAM/MUC18 in this aspect.

\section{The role of METCAM/MUCI 8 in the progression of ovarian cancer}

Aldovini et al. ${ }^{19}$ first showed that METCAM/MUC18 expression is significantly associated with advanced stage tumors, and serous and undifferentiated subtypes, and is a marker stronger than residual disease in predicting early tumor relapse and independent marker of poor prognosis for epithelial ovarian cancer. ${ }^{19}$ Our recent report also supported the notion that METCAM/MUC18 expression is correlated with the progression of ovarian cancer. ${ }^{20}$ Furthermore, Wu et al. ${ }^{41}$ reported that METCAM/MUC18 expression is high in metastatic ovarian cancers in comparison with other pathological types of ovarian epithelial tissues. From in vitro studies by using siRNAs to silence the endogenous METCAM/MUC18 expression in the SKOV-3 cell line, they showed that decreasing endogenous METCAM/ MUC18 expression in the cells increases apoptosis and cell spreading and invasion, suggesting that METCAM/MUC18 may play a positive role in the progression of ovarian cancer cells. ${ }^{41}$ However, this notion has not been directly supported by evidence from animal studies.

\section{METCAM/MUCI 8 is expressed at a lower level in the ovarian cancer cell lines established from malignant ascites than in the cell lines from adenocarcinomas}

To test if he above notion is correct or simply a fortuitous correlation, we directly tested the role of METCAM/MUC18 in the progression of epithelial ovarian cancer in vitro and in vivo. First, we re-evaluated the expression of METCAM/MUC18 in one immortalized normal ovarian epithelial cell line (IOSE) and five human ovarian cancer cell lines, BG-1, HEY, CAOV-3, SK-OV-3 and NIHOVCAR $3,{ }^{42}$ as shown in the following Figure 2.

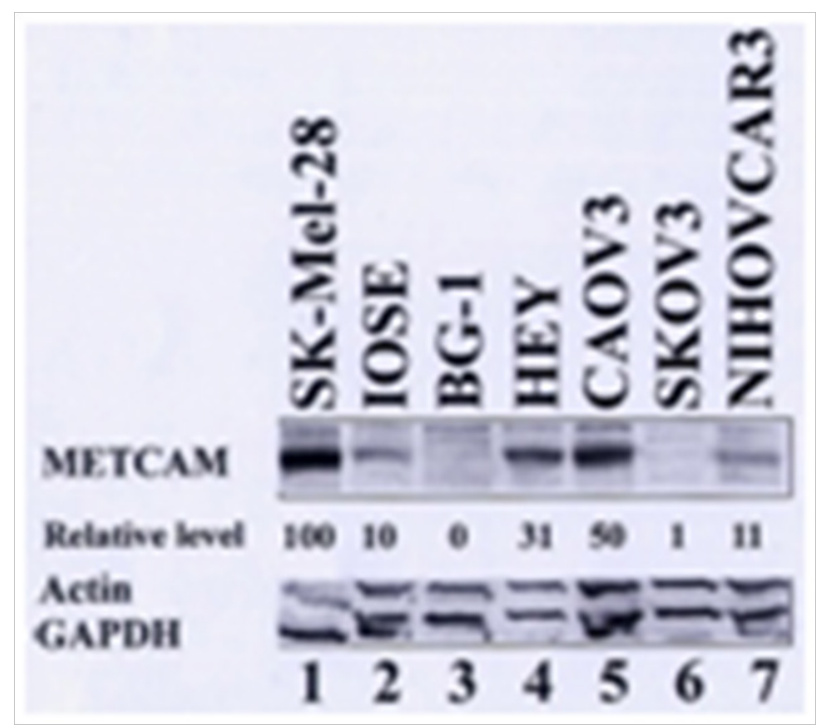

Figure 2 Expression of METCAM in various human ovarian cancer cell lines. The expression of METCAM/MUCI 8 in the lysates from various cells lines was determined by Western blot (WB) analysis. Cell lysate from a human melanoma cell line, SK-Mel-28, was used as a positive control (lane I) and those from human ovarian cancer cell lines, BG-I (lane 3) and SK-OV-3 (lane 6) as negative controls. METCAM/MUCI 8 expression in cell lysates from one immortalized human ovarian epithelial cells (IOSE) and in five human ovarian cancer cell lines is shown in lanes 2 to 7.The number under each lane indicates the relative level of METCAM/MUCI 8 of each cell line, assuming that in SKMel-28 as $100 \%$. Only the house-keeping genes, actin and GAPDH, are shown here as the loading controls.

As shown in the above Figure 2, the expression level of METCAM/MUC18 in one immortalized normal ovarian epithelial cell line (IOSE) was about $10 \%$ and that in five ovarian cancer cell lines, BG-1, HEY, CAOV-3, SK-OV-3 and NIHOVCAR3, ranged from zero to $50 \%$ (assuming that a positive control, human melanoma cell line SK-Mel-28, expressed 100\% of METCAM/MUC18). Since METCAM/MUC18 was expressed at a level of $31-50 \%$ in two out of three cell lines established from primary adenocarcinomas (HEY and CAOV3), but poorly expressed (1-11\%) in two cell lines established 
from malignant ascites (SKOV3 and NIHOVCAR3), it appeared that METCAM/MUC18 was expressed poorer in malignant cell lines than in primary adenocarcinomas, suggesting that METCAM/MUC18 may play a negative role in the progression of ovarian cancer. The above result also provided an important information for us to possibly choose two ovarian cancer cell lines, BG-1 (established from a poorly differentiated adenocarcinoma) and SK-OV-3 (established from an adenocarcinoma metastasis as malignant ascites), which expressed very low levels of METCAM/MUC18 (zero and 1\%, respectively), for in vitro and in vivo studies. We included only the results from the studies by using the cell line, SK-OV-3, than those by using the cell line, BG-1, because the BG-1 cell line in some laboratories has been reported to be contaminated with the breast cancer cell line MCF7. ${ }^{43}$

Two complementary methods are commonly used to biochemically alter the expression of METCAM/ MUCI 8 in the cell lines

To determine the effect of a specific gene on cellular behaviors, two complementary methods are commonly used to alter the expression of a gene in cells:

a. Enforced expression of a gene in cell lines that did not or weakly express the protein ${ }^{35}$ and

b. siRNA (small interference RNA)-knockdown expression of the gene in cell lines that endogenously express the protein. ${ }^{44}$

The clones, which highly expressed the protein or siRNA, were isolated, and used for the in vitro and in vivo studies.

\section{METCAM/MUC 8 expression in G4 I 8R-clones derived from the SK-OV-3 cell line}

Since SK-OV-3 cell line did not or weakly express METCAM/ MUC18, to determine if METCAM/MUC18 expression affects the in vitro and in vivo cellular behaviors of the cells, only the enforced expression method was used to increase the expression of the gene in this cell line. All the G418R-clones should express METCAM/ MUC18, albeit at different levels in different clones. The control cells, which were transfected with the empty vector that did not contain the human METCAM/MUC18 cDNA, were also G418R and should not express METCAM/MUC18, similar to the parental SK-OV-3 cells. Figure 3 shows the expression of METCAM/MUC18 in the two typical G418R clones was higher than the empty-vector control clone derived from SK-OV-3 cell line. ${ }^{42}$

The above G418R clones from the SK-OV-3 cell line are then used to study effects of over-expression of the METCAM/MUC18 gene on their in vitro cellular behaviors, such as cellular motility and invasiveness, and on in vivo tumorigenesis and metastasis in animal models.

Over-expression of METCAM/MUCI8 decreased epithelial-to-mesenchymal transition (EMT) of SKOV-3 cells

Epithelial-to-mesenchymal transition (EMT) is a biological process by which carcinoma cells (cancer cells derived from epithelial cells) detach from the surrounding tissue and acquire characteristics of mesenchymal cells, which are unique motile and spindle-shaped cells with end-to-end polarity ${ }^{45}$. Cells that have undergone EMT can migrate out of their epithelial layers to distant organs, at where they may remain mesenchymal or re-differentiate into epithelial cells by a process known as mesenchymal-to-epithelial transition (MET). Thus
EMT may be a process pre-required for tumor progression. In addition to increased motility, carcinoma cells via EMT may become stem celllike, protected from senescence, apoptosis and immune surveillance, and resistant to conventional and targeted therapies. ${ }^{45}$ The degree of EMT in cells usually can be determined by the extent of motility and invasiveness of the cells in vitro. The above G418R clones were used to determine the effects of enforced expression of METCAM/MUC18 on their in vitro motility and invasiveness.

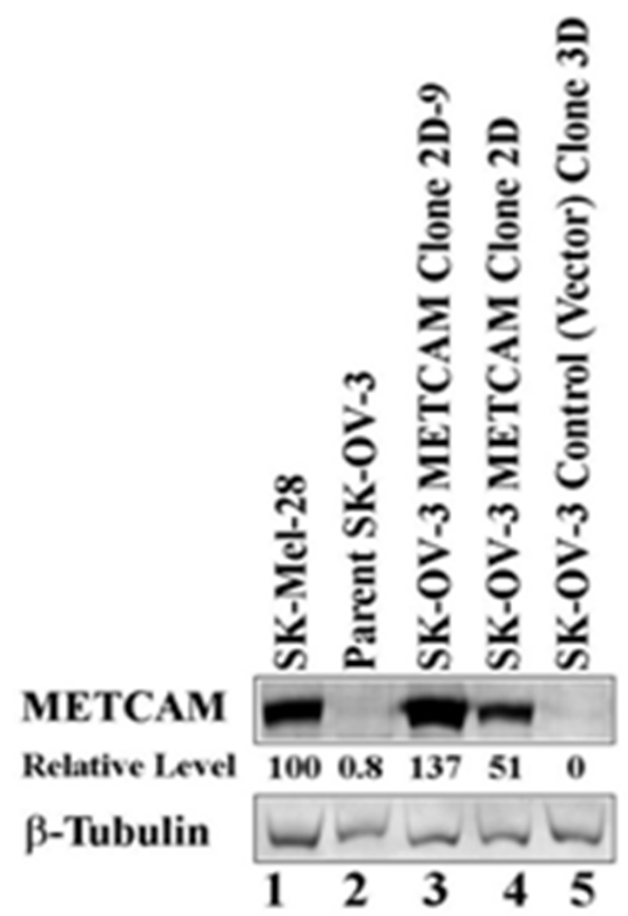

Figure 3 Human METCAM/MUCI8 expression in lysates prepared from various clones/cells. METCAM/MUCI 8 expression in the cell lysate from a human melanoma cell line, SK-Mel-28, was used as a positive control (lane I) and that from the parental human ovarian cancer cell line, SK-OV-3, as a negative control (lane 2). METCAM/MUCI 8 expression in cell lysates from one single SK-OV-3 clone (METCAM Clone 2D-9) and two pooled SK-OV-3 clones (METCAM Clone 2D and Control (Vector) Clone 3D) are shown in lanes 3-5. Both the METCAM Clone 2D-9 and the METCAM Clone 2D were derived from SK-OV-3 cells transfected with the human METCAM/MUCI8 cDNA gene. The Control (Vector) Clone 3D was from SK-OV-3 cells which were transfected with the empty vector.The number under each lane indicates the relative level of METCAM/MUCI 8 of each cell line, assuming that in SKMel-28 as $100 \%$. b-tubulin is shown as the loading control.

If METCAM/MUC18 plays a positive role in mediating EMT in this cell line, we should observe an increased motility and invasiveness of these stable clones that overly express METCAM/MUC18. On the contrary, if it plays a negative role in EMT, we should observe a decreased motility and invasiveness of these stable clones that overly express METCAM/MUC18, which indeed were observed in Figure 4. From the results, we strongly suggested that huMETCAM/MUC18 plays a negative role in the EMT of SK-OV-3 cells and METCAM/ MUC18 directly causes the decreased EMT of SK-OV-3 cells.

\section{Over-expression of METCAM/MUCI 8 suppressed in vivo tumorigenesis and the malignant progression of the human ovarian cancer cell line SK-OV-3}

To scrutinize the notion that METCAM/MUC18 may play a positive role in the development of ovarian cancer, ${ }^{41}$ we used the above 
clones to perform studies in model animals. ${ }^{42}$ We determined effects of METCAM/MUC18 over-expression on in vivo tumorigenicity of SKOV3 cells in female nude mice after SC injection at either dorsal (DSC) or ventral (VSC) side. As shown in Figure 5 (left panel) that tumor proliferation of the METCAM clone 2D was much lower than that of the control (vector) clone at both sites, indicating that overexpression of METCAM/MUC18 decreased tumorigenicity of SKOV-3 cells in nude mice. Consistent with the results in Figure 5 (left panel), Figure 5 (right panel) shows that final tumor weights of the METCAM clone 2D were also lower than those of the control (vector) clone 3D at both sites, indicating that over-expression of METCAM/ MUC18 decreased the final tumor weights of SK-OV-3 cells in nude mice. ${ }^{42}$
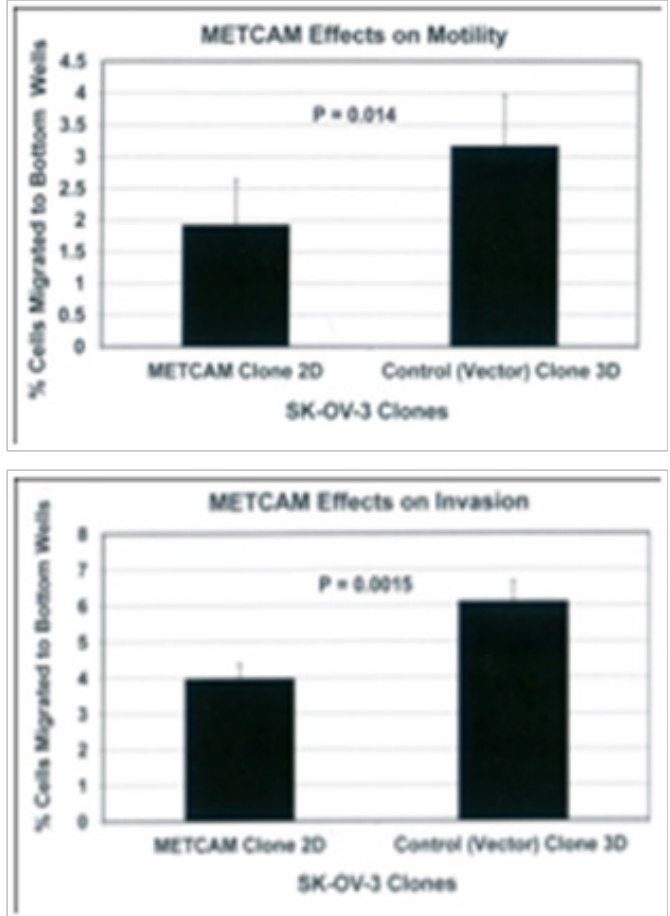

Figure 4 Effects of METCAM/MUCI8 expression on the motility (left panel) and invasiveness (right panel) of SK-OV-3 cell line. (Left panel) For the motility test, the METCAM clone 2D and the Control (Vector) clone 3D of SK-OV-3 cells were used. Six hours after seeding to the top wells, cells migrating to the bottom wells were determined. Means and standard deviations of triplicate values of the motility tests are indicated. $P$ value, which was determined by analyzing two sets of data with the Student's $t$ test by using the one-tailed distribution-type 2 method, was 0.014 , indicating that the result is statistically different. (Right panel) For invasiveness test, the METCAM clone 2D and the Control (Vector) clone 3D of SK-OV-3 cells were used. Six hours after seeding cells to the top wells, cells migrating to the bottom wells were determined Means and standard deviations of triplicate values of the invasiveness tests are indicated. P value, which was determined by analyzing two sets of data with the Student's $t$ test by using the one-tailed distribution-type 2 method, was 0.0015 , indicating that the result is statistically different.

Taken together, we conclude that over-expression of METCAM/ MUC18 suppressed in vivo tumorigenesis of SK-OV-3 cells at nonorthotopic (ventral and dorsal) subcutaneous sites in nude mice. Furthermore, the tumors induced by the METCAM clone 2D were confined to small regions, as shown in the results of $\mathrm{H} \& \mathrm{E}$ and $\mathrm{IHC},{ }^{42}$ whereas the tumors induced by the control (vector) clone 3D developed serious tumors, suggesting that tumors from the 2D clone appeared to be dormant; thus METCAM/MUC18 may function similarly to other tumor/metastasis suppressors in other tumor cells. ${ }^{46}$
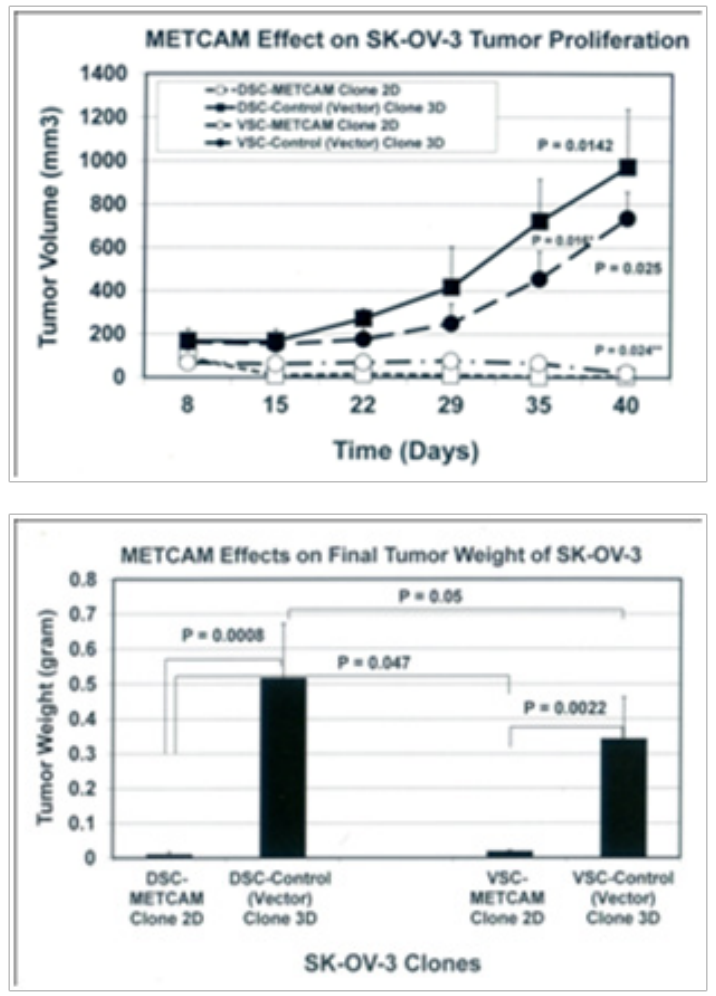

Figure 5 Effect of over-expression of huMETCAM/MUCI8 on the tumor proliferation (left panel) and final tumor weight (right panel) at S.C. sites of human ovarian cancer SK-OV-3 clones. The pooled 2D clone expressed $51 \%$ of METCAM, whereas the pooled 3D clone (Vector) expressed $0 \%$ of METCAM.

To further determine the effect of METCAM/MUC18 overexpression on in vivo tumorigenicity of SK-OV-3 cells in the orthotopic site (intraperitoneal (IP) cavity), SK-OV-3 cells from the pooled METCAM clone 2D and the control (vector) clone 3D were IP injected into female nude mice. The mice in the control group, which were injected with the control vector clone $3 \mathrm{D}$, developed swollen abdominal cavity and tumors, but not the mice in the test group, which were injected with the METCAM clone 2D. Consistent with the observation, the final weights of abdominal tumors, as shown in Fig. 6 (left panel), and volumes of ascites were measured, as shown in Figure 6 (right panel), were significantly larger in the group injected with the control vector clone 3D than those injected with the METCAM/MUC18-expressing clone 2D. We concluded that over-expression of METCAM/MUC18 suppressed the tumorigenicity and ascites formation of SK-OV-3 cells in IP cavities in nude mice. ${ }^{42}$

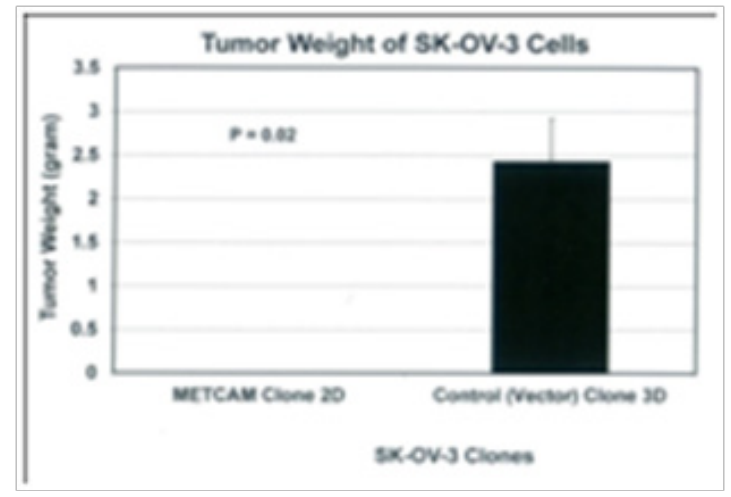




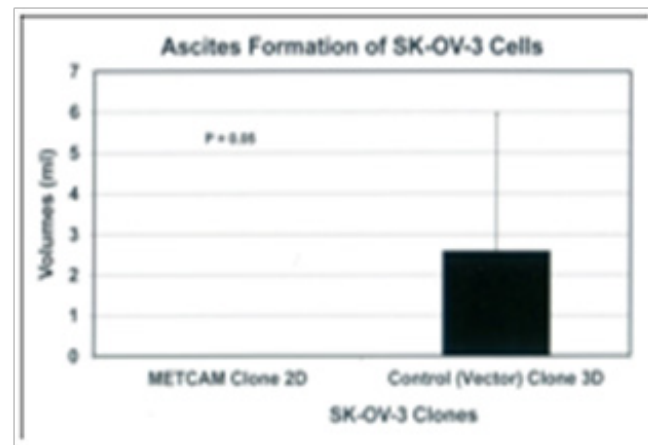

Figure 6 Effect of over-expression of huMETCAM/MUCI 8 on the final weight of solid tumors (left panel) and final ascites volumes of ascites formed in the intraperitoneal cavity (right panel) at orthotopic sites (the intraperitoneal cavity) from human ovarian cancer SK-OV-3 clones. The pooled 2D clone expressed $5 \mathrm{I} \%$ of METCAM, whereas the pooled 3D clone (Vector) expressed $0 \%$ of METCAM.

Taken together, METCAM/MUC18 expression in SK-OV-3 cells decreased the tumor proliferation and tumorigenesis at $\mathrm{SC}$ sites as well as at the orthotopic IP site, strongly suggesting that METCAM/ MUC18 is a novel tumor and metastasis suppressor for the progression of human ovarian cancer cells.

\section{Preliminary mechanisms of METCAM/MUCI 8- mediated suppression of the progrssion of SK-OV-3 cells}

Mechanisms of METCAM/MUC18-mediated suppression of the progression of human ovarian cancer cells have not been studied. By deducing knowledge learned from METCAM/MUC18-induced tumorigenesis of other tumor cell lines, such as, melanoma, cancers in breast and prostate and nasopharyngeal carcinoma, METCAM/ MUC18 may affect tumorigenesis by cross-talk with many downstream signaling pathways that regulate proliferation, survival pathway, apoptosis, metabolism, and angiogenesis of tumor cells., ${ }^{70}$ To investigate if METCAM/MUC18-mediated tumor suppression also affected expression of its downstream effectors, such as indexes of apoptosis/anti-apoptosis, proliferation, survival, aerobic glycolysis, and angiogenesis, we determined the expression of levels of $\mathrm{Bcl} 2$, Bax, PCNA, LDH-A, pan-AKT, phospho-AKT(Ser 473), and the ratio of phospho-AKT/AKT in tumor lysates by using Western blot analyses. ${ }^{42}$ From the results, we suggest that over expression of METCAM/MUC18 may suppress tumorigenesis and malignant progression of ovarian cancer cells in nude mice by decreasing their abilities in proliferation, aerobic glycolysis, and angiogenesis via decreasing the absolute levels of pan-AKT and phospho-AKT, but not altering the apoptosis/anti-apoptosis and survival pathways. ${ }^{42}$ This is consistent with the results from clinical specimens. ${ }^{20}$

\section{Discussion}

The above conclusion contradicts the results of a positive correlation of clinical prognosis with the increased expression of METCAM/MUC18 in malignant ovarian cancer specimens..$^{19,20,41}$ This suggests that the positive correlation in this case is fortuitous and that we should not assume a positive role of METCAM/MUC18 in the progression of ovarian cancer without the support of tests in an animal model. Our results also contradict the previously established notion that METCAM/MUC18 serves as a tumor promoter in both prostate cancer cells and breast cancer cells, and as a metastasis promoter in human melanoma cells, prostate cancer, and breast cancer. ${ }^{30,35-40}$
The role of METCAM/MUC18 as a tumor suppressor was not only conclusively demonstrated in the human ovarian cancer cell line, SK$\mathrm{OV}-3,{ }^{42}$ but also in a mouse melanoma cell line, K1735-947 and one NPC cell line, NPC-TW $01^{34,48}$ (Wu, unpublished results). METCAM/ MUC18 has also been demonstrated as a metastasis suppressor in the two human ovarian cancer cell lines, SK-OV-3 cells and BG-1 cells ${ }^{42}(\mathrm{Wu}$, unpublished results). Thus sufficient evidence is provided to support the novel suppressor role of METCAM/MCU18 in the progression of human ovarian epithelial cancers.

The most intriguing, unique biological function of METCAM/ MUC18 in tumorigenesis and metastasis is that it seems to play a dual role in the progression of some tumor cell lines. ${ }^{49}$ It is not clear why METCAM/MUC18 plays a dual role in tumorigenicity and metastasis. One point is clear, which is that METCAM/MUC18 plays an opposite role in different cancer types or in different clones/ sublines of the same cancer type. ${ }^{49}$ Thus it is logical to propose that the effect of METCAM/MUC18 on the progression of epithelial cancers is modulated by different intrinsic factors in different tumor cells/types. The dual role of METCAM/MUC18 is very likely due to the presence of different interacting partners intrinsic to each cancer cell type and different clone, or perhaps due to different heterophilic ligands, which unfortunately have not been identified. ${ }^{30,49}$ Interactions of METCAM/MUC18 with different sets of intrinsic partners may result in the promotion or suppression of tumorigenicity and metastasis via increasing or decreasing aerobic glycolysis, proliferation, angiogenesis, other growth-promoting pathways, as well as altering tumor cell motility, invasiveness, and vascular metastasis.

The tumor/metastasis suppressor role of human METCAM/ MUC18 in the progression of human ovarian cancer cells may point to the possibility that METCAM/MUC18 may induce tumor dormancy. How METCAM/MUC18 affects tumor dormancy should be an interesting aspect for future investigation, since tumor dormancy may be due to intrinsic growth inhibition, immunological suppression, and/or angiogenic suppression. ${ }^{50}$

\section{Perspectives and clinical applications}

The tumor suppressor role of METCAM/MUC18 in the progression of human ovarian cancer cell lines may be useful for clinical application. Indeed many tumor and metastatic suppressors, such as KISS1, KAI1, nm23, MAP2K4, and some microRNA have been used for clinical applications. ${ }^{51}$ Three strategies have been developed, such as

i. Reconstitution of suppressor genes by induction of the endogenous locus or by gene therapy,

ii. Direct administration of the suppressor proteins,

iii. Targeting essential downstream pathways that are activated by loss of suppressor function.

In light of these, METCAM/MUC18 cDNA gene may be used for gene therapy by using the adenovirus-associated virus vector or a replication-defective adenovirus. The recombinant METCAM/ MUC18 protein, or METCAM-derived peptides, or small molecule mimetics of METCAM may also be used directly. The recombinant cognate ligand of METCAM/MUC18 may potentially be used also. However many downstream pathways of METCAM/MUC18 may not be useful because METCAM/MUC18 appeared to involve many of them. The above strategies may be used for clinical treatments by keeping ovarian cancer cells in a dormant state or arresting the cancer cells at the stage of micro-metastases. 


\section{Conclusion}

In summary, we provided evidence to show that METCAM/ MUC18 is a novel suppressor for the tumorigenesis and malignant progression of the human ovarian cancer SK-OV-3 cells:

A. METCAM/MUC18 was expressed at lower levels in malignant cell lines than in primary adenocarcinomas, suggesting that METCAM/MUC18 may play a negative role in the progression of ovarian cancer.

B. A high expression level of METCAM/MUC18 inhibits the EMT of SKOV3 cancer cells.

C. METCAM/MUC18 expression inhibited the tumorigenicity at the subcutaneous sites as well as the tumorigenicity and ascites formation in the intra-peritoneal cavity of an athymic nude mouse model.

Since the METCAM/MUC18 expressed in the tumors and ascites cells were similar to that in the injected clones/cells, the protein was not modified to manifest these processes. Taken together, we conclude that METCAM/MUC18 serves as a tumor suppressor as well as a metastasis suppressor for the human ovarian cancer SK-OV-3 cells. METCAM/MUC18 may suppress tumorigenesis and malignant progression of ovarian cancer cells in nude mice by decreasing their abilities in proliferation, aerobic glycolysis (metabolism), and angiogenesis via down-regulating the PI3K-AKT signaling pathway.

\section{Acknowledgements}

We thank the financial supports from Emory University School of Medicine (USA), Chung Yuan Christian University, and grants from NSC (NSC-101-2320-B-033-001 and -003), Taiwan (GJW).

\section{Conflicts of interest}

None.

\section{References}

1. Siegel R, Ma J, Zou Z, Jemal A. Cancer statistics 2014. CA Cancer J Clin. 2014;64(1):9-29.

2. Clarke-Pearson DL. Screening for ovarian cancer. $N$ Engl $J$ Med. 2009;361:170-177.

3. Wei W, Dizon D, Vathipadiekal V, et al. Ovarian cancer: genomic analysis. Ann Oncol. 2013;24(Suppl 10):x7-x15.

4. Devouassoux-Shisheboran M, Genestie C. Pathobiology of ovarian carcinomas. Chinese J Cancer. 2015;34(1):50-55.

5. Menon U, Griffin M, Gentry-Maharaj A. Ovarian cancer screeningcurrent status, future directions. Gynecol Oncol. 2014;132(2):490-495.

6. Jacob F, Nixdorf S, Hacker NF, et al. Reliable in vitro studies require appropriate ovarian cancer cell lines. J Ovarian Res. 2014;7:60

7. Harahan D, Weinberg RA. Hallmarks of cancer: the next generation. Cell. 2011;144(5):646-674.

8. Alberts B, Johnson A, Lewis J, et al. Chapter 19: Cell junctions, cell adhesion, and the extracellular matrix. Molecular Biology of the Cell, (5th edn), Garland Science, Taylor \& Francis Group, LLC, New York, USA and Abingdon, UK. 2008;pp.1131-1195.

9. Cavallaro U, Christofori G. Cell adhesion and signaling by cadherins and Ig-CAMs in cancer. Nat Rev Cancer. 2005;4(2):118-132.

10. Bellone S, Siegel ER, Cocco E, et al. Over-expression of epithelial cell adhesion molecule in primary, metastatic, and recurrent/chemotherapyresistant epithelial ovarian cancer: implications for epithelial cel adhesion molecule-specific immunotherapy. Int J Gynecology Cancer. 2009;19(5):860-866.

11. Rump A, Morikawa Y, Tanaka M, et al. Binding of ovarian cancer antigen CA125/MUC16 to mesothelin mediates cell adhesion. $J$ Bio Chem. 2004;279(10):9190-9198.

12. Villegas-Pineda JC, Garibay-Cerdenares OL, Hernández-Ramírez VI, et al. Integrins and haptoglobin: Molecules overexpressed in ovarian cancer. Pathol Res Pract. 2015;211(12):973-981.

13. Bourguignon LYW, Gilad E, Rothman K, et al. Hyaluronan-CD44 interaction with IQGAP1 promotes Cdc42 and ERK signaling, leading to actin binding, Elk-1/estrogen receptor transcription activation, and ovarian cancer progression. J Biol Chem. 2005;280(12):11961-11972.

14. Euer NI, Kaul S, Deissler H, et al. Identification of L1CAM, Jagged2, and neuromedin $\mathrm{U}$ as ovarian cancer-associated antigens. Oncology Rep. 2005;13(3):375-387.

15. Imai T, Horiuchi A, Shiozawa $T$, et al. Elevated expression of $\mathrm{E}-$ cadherin and alpha-, beta-, and gamma-catenins in metastatic lesions compared with primary epithelial ovarian carcinomas. Human Pathol. 2004;35(12):1469-1476.

16. Morin PJ. Claudin proteins in ovarian cancer. Disease Markers 2007;23(5-6):453-457.

17. Pietzner K, Woopen H, Richter R, et al. Expression of EpCAM in paired tumor samples of patients with primary and recurrent serous ovarian cancer. Int J Gynecology Cancer. 2013;23(5):797-802.

18. Rosso O, Piazza T, Bongarzone I, et al. The ALCAM Shedding by the Metalloprotease ADAM17/TACE Is Involved in Motility Ovarian Carcinoma Cells. Mol Cancer Res. 2007;5(12):1246-1253.

19. Aldovini D, Demichelis F, Doglioni C, et al. M-CAM expression as marker of poor prognosis in epithelial ovarian cancer. Int $J$ Cancer. 2006;119(8):1920-1926

20. Wu GJ, Dickerson EB. Frequent and increased expression of human METCAM/MUC18 in cancer tissues and metastatic lesions associates with the clinical progression of human ovarian carcinoma. Taiwan $J$ Obstet Gynecol. 2014;53(4):509-517.

21. Ponnusamy MP, Lakshmanan I, Jain M, et al. MUC4 mucin-induced epithelial to mesenchymal transition: a novel mechanism for metastasis of human ovarian cancer cells. Oncogene. 2010;29(42):5741-5754.

22. Wang H, Tan M, Zhang S, et al. Expression and Significance of CD44, CD47 and c-met in Ovarian Clear Cell Carcinoma. Int J Mol Sci. 2015;16(2):3391-3404.

23. Altevogt P, Doberstein K, Fogel M. L1CAM in human cancer International J Cancer. 2015;138(7):1565-1571.

24. Vieira, AF, Paredes J. P-cadherin and the journey to cancer metastasis. Mol Cancer. 2015;14:178

25. Kaur S, Kenny HA, Jagadeeswaran S, et al. b3-Integrin Expression on Tumor Cells Inhibits Tumor Progression, Reduces Metastasis, and Is Associated with a Favorable Prognosis in Patients with Ovarian Cancer Am J Pathology. 2009;175(5):2184-2196.

26. Qiu X, Cheng JC, Klausen C, et al. Transforming growth factor-a induces human ovarian cancer cell invasion by down-regulating E-cadherin in a Snail-independent manner. Biochem Biophys Res Communications. 2015;461:128-135.

27. Lin $X$, Shang $X$, Manorek G, et al. Regulation of the EpithelialMesenchymal Transition by Claudin-3 and Claudin-4. PLOS. 2013;One 8(6):e67496. 
28. Fan Q, Cheng JC, Qiu X, et al. EpCAM is up-regulated by EGF via ERK1/2 signaling and suppresses human epithelial ovarian cancer cell migration. Biochem Biophy Res Comm. 2015457(3):256-261.

29. Upheber S, Karle A, Miller J, et al. Alternative splicing of KAI1 abrogates its tumor-suppressive effects on integrin $\alpha \mathrm{v} \beta 3$-mediated ovarian cancer biology. Cell Signal. 2015;27(3):652-662.

30. Wu GJ. METCAM/MUC18 expression and cancer metastasis. Current Genomics. 2005;18(6):333-349.

31. Lehmann JM, Reithmuller G, Johnson JP. MUC18, a marker of tumor progression in human melanoma. Proc Natl Acad Sci USA. 198986(24):9891-9895.

32. Shih IM. The role of CD146 (Mel-CAM) in biology and pathology. $J$ Pathol. 1999;189(1):4-11.

33. Shih IM, Hsu M-Y, Palazzo JP, Herlyn M. The cell-cell adhesion receptor MEL-CAM acts as a tumor suppressor in breast carcinoma. Am J Pathol. 1997;151(3):745-751.

34. Lin JC, Chiang CF, Wang SW, et al. Significance of expression of human MECAM/MUC18 in nasopharyngeal carcinomas and metastatic lesions. Asian Pac J Cancer Prev. 2014;15(1):245-252.

35. Wu GJ, Wu MWH, Liu Y. Enforced expression of human METCAM/ MUC18 increases the tumorigenesis of human prostate cancer cells in nude mice. J Urology. 2011;185(4):1504-1512.

36. Zeng G, Cai S, Wu GJ. Up-regulation of METCAM/MUC18 promotes motility, invasiveness and tumorigenesis of human breast cancer cells. BMC Cancer. 2011;11:113.

37. Zeng G, Cai S, Liu Y, et al. METCAM/MUC18 augments promotes migration, invasion and tumorigenicity of human breast cancer SKBR-3 Cells. Gene. 2012;492(1):229-238.

38. Wu GJ, Fu P, Wang S-W, et al. Enforced expression of MCAM/MUC18 increased in vitro motility and invasiveness and in vivo metastasis of two mouse melanoma K1735 Sublines in a syngeneic mouse model. Mol Cancer Res. 2008;6(11):1666-1677.

39. Wu GJ, Qiong P, Fu P, et al. Ectopical expression of human MUC18 increases metastasis of human prostate cancer cells. Gene. 2004;327(2):201-213.
40. Zeng Q, Li W, Lu D, et al. CD146, an epithelial-mesenchymal transition inducer, is associated with triple-negative breast cancer. Proc Natl Acad Sci USA. 2012;109(4):1127-1132.

41. $\mathrm{Wu} \mathrm{Z}, \mathrm{Wu} \mathrm{Z}, \mathrm{Li}$, et al. MCAM is a novel metastasis marker and regulates spreading, apoptosis and invasion of ovarian cancer cells. Tumor Biol. 2012;33(5):1619-1628.

42. Wu GJ, Zeng G. METCAM/MUC18 is a novel tumor and metastasis suppressor for the human ovarian cancer SKOV3 cells. BMC Cancer. $2016 ; 16: 136$.

43. Li Y, Arao Y, Hall JM, et al. Research Resource: STR DNA Profile and Gene Expression Comparisons of Human BG-1 Cells and a BG-1/ MCF-7 Clonal Variant. Mol Endocrinol. 2014;28(12):2072-2081.

44. Wu GJ. Human METCAM/MUC18 as a novel biomarker to drive and its specific SiRNAs to block the malignant progression of prostate cancer. $J$ Cell Sci Therapy. 2015;6:5(1000227).

45. Hugo H, Ackland ML, Blick T, et al. Epithelial-mesenchymal and mesenchymal-epithelial transitions in carcinoma progression. $J$ Cell Physiology. 2007;231(2):374-383.

46. Wikman H, Vessella R, Pantel K. Cancer micrometastasis and tumor dormancy. APMIS. 2008;116(7-8):754-770.

47. Wu GJ. Over-expression of METCAM/MUC18 suppresses tumorigenesis and metastasis of mouse melanoma K1735-9 cells in syngeneic mice. Clinical \& Experimental . 2016;33(8):817-828.

48. Liu YC. The putative role of human METCAM/MUC18 in modulating the development and progression of nasopharyngeal carcinoma. M.S. thesis under the supervision of Dr. Guang-Jer Wu, Department of Bioscience Technology, Chung Yuan Christian University, Chung Li, Taiwan. 2014

49. Wu GJ. Dual roles of METCAM in the in the progression of different cancers. J Oncology 2012: 853797. 2012

50. Aguirre-Chiso JA.Models, mechanisms and clinical evidence for cancer dormancy. Nat Rev Cancer. 2007;7(11):834-846.

51. Smith SC, Theodorescu D. Learning therapeutic lessons from metastasis suppressor proteins. Nat Rev Cancer. 2009;9(4):253-264. 